\title{
SCATTERED LIGHT FROM ENVELOPES AROUND N-TYPE STARS
}

\author{
BENGT GUSTAFSSON AND KJELL ERIKSSON \\ Uppsala Astronomical Observatory \\ Uppsala, Sweden \\ DAN KISELMAN
}

Royal Academy of Sciences, Stockholm Observatory

Saltsjöbaden, Sweden

NILS OLANDER

Dept. of Physics and Mathematics, MidSweden University

Sundsvall, Sweden

HANS OLOFSSON

Stockholm Observatory

Saltsjöbaden, Sweden

AND

HUGO E. SCHWARZ

Nordic Optical Telescope

Santa Cruz de La Palma, Canary Islands, Spain

\begin{abstract}
Circumstellar emission in the $\mathrm{NaI}$ and $\mathrm{KI}$ resonance lines has been detected from three carbon stars using high-resolution spectroscopy. Some properties of the circumstellar envelopes are discussed.
\end{abstract}

Circumstellar envelopes around three bright $\mathrm{N}$-type stars, R Scl, X TrA, and $\mathrm{V} \mathrm{Aql}$, have been detected in emissions in resonance lines from $\mathrm{K} I$ and $\mathrm{Na}$ I. This radiation, most probably scattered photospheric radiation, was discovered using high-resolution spectroscopy.

From the observations of the K I $769.9 \mathrm{~nm}$ emission we find systemic and expansion velocities in fair agreement with those obtained from the $\mathrm{CO}$ millimeter lines. We find a decline of the surface brightness of the scattered light as a function of the angular distance from the star, $\beta$, of approximately $\beta^{-3}$, in agreement with the assumption of optically thin emission and constant expansion velocity, mass-loss rate, and $\mathrm{KI}$ abundance. 


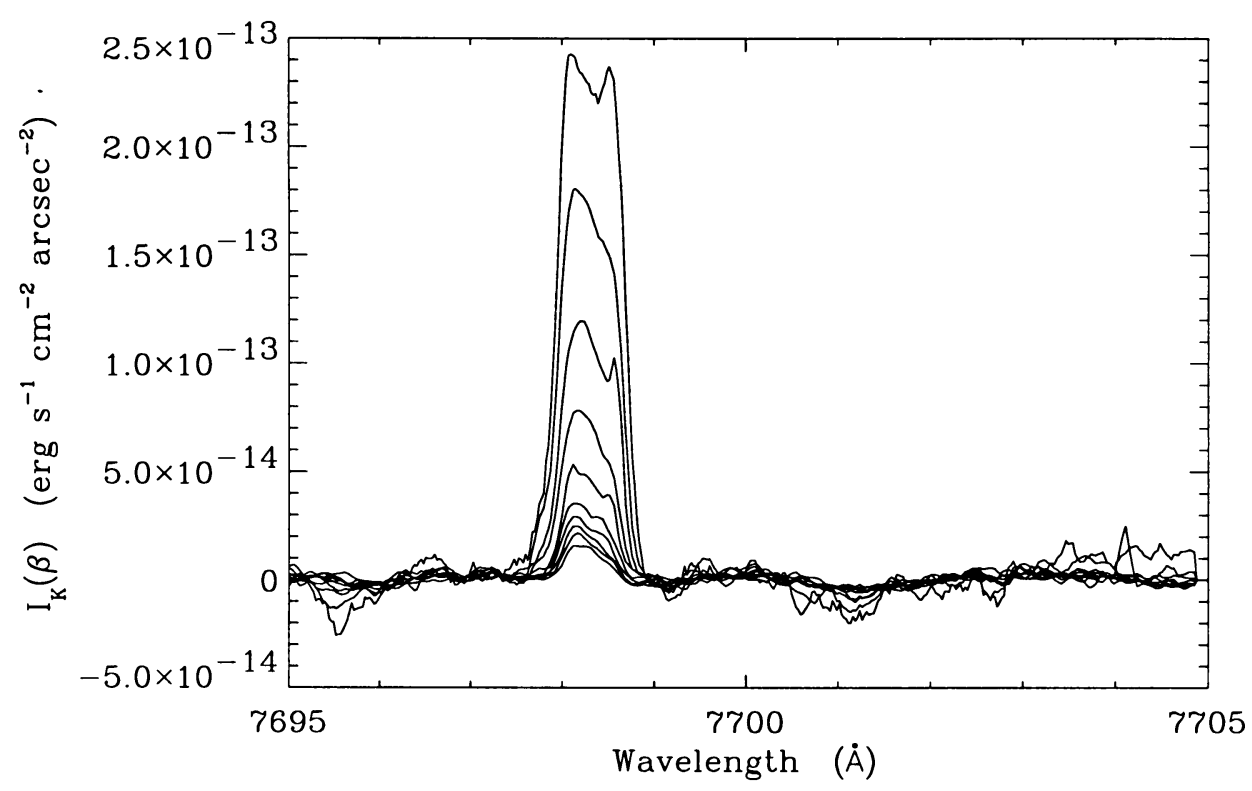

Figure 1. Subtracted K I spectra (off-star minus scaled on-star) for different distances from $\mathrm{R}$ Scl. The off-star spectra were obtained with the star approximately $4^{\prime \prime}$ outside the slit, in the slit direction. The spacing between successive spectra is $0 . " 83$.

Our mass-loss rate estimates from the $\mathrm{KI}$ line emissions agree rather well with those obtained from $\mathrm{CO}$, which suggests that a considerable fraction of the potassium stays neutral throughout the envelope. If ionization of potassium from interstellar ultraviolet radition as well as from some assumed chromospheric fluxes is considered, the mass-loss rates increase by one order of magnitude. This puts strong upper limits on the photoionizing chromospheric UV emission from these stars. Details of this work are presented in Gustafsson et al. (1997).

Optical imaging in $5 \mathrm{~nm}$ wide $\mathrm{Na}$ and $\mathrm{K}$ filters shows stellar envelopes around the "detached CO shell stars" R Scl, U Ant, and S Sct, with envelope diameters between $20^{\prime \prime}$ and $2^{\prime}$ and a flat brightness distribution. These diameters and the morphology are remarkably similar to those of maps of $\mathrm{CO} \mathrm{mm}$ line emission of the objects. The optical images were obtained at the ESO 3.6-m telescope using a coronographic polarimetry technique to increase the contrast between faint envelopes and the stellar light scattered in the terrestrial atmosphere.

The optical images probably show general dust-scattered light, and the CO maps reflect the morphology of the molecular line emission, while the spectra represent the true resonance-scattering envelope. 


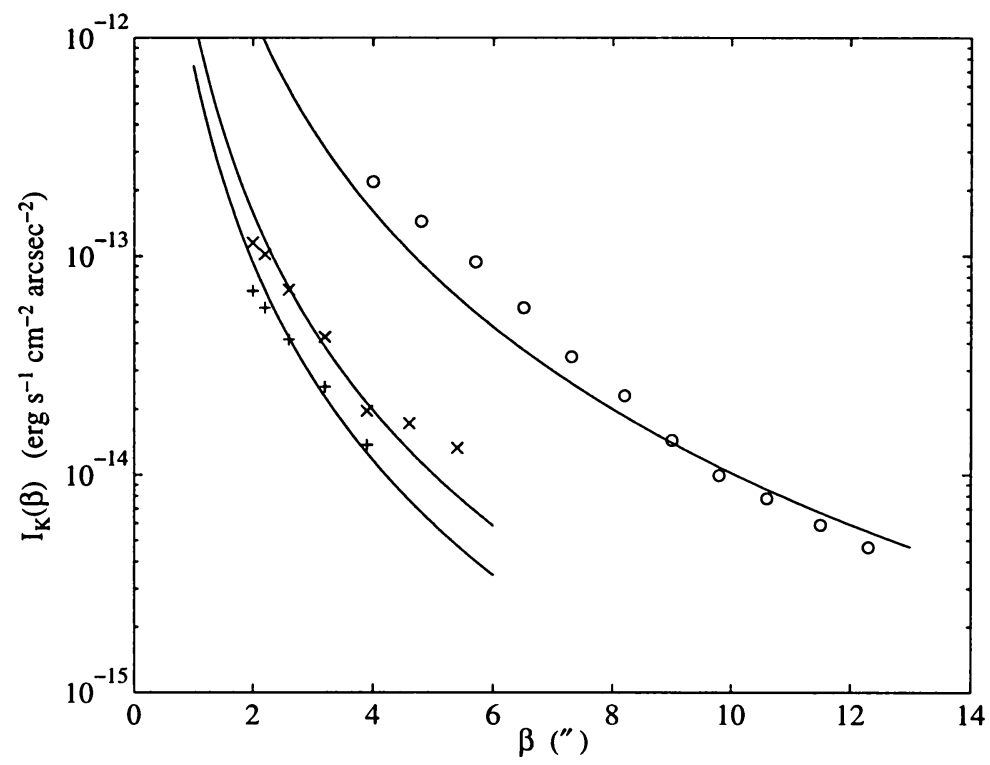

Figure 2. The wavelength-integrated circumstellar $\mathrm{KI}$ emission as a function of angular distance $(\beta)$ to the stars $\mathrm{R} \mathrm{Scl}(\mathrm{o}), \mathrm{X} \operatorname{TrA}(\mathrm{x})$ and $\mathrm{V}$ Aql $(+)$. The curves show $\beta^{-3}$ fits to the data.

\section{References}

Gustafsson, B., Eriksson, K., Kiselman, D., Olander, N. \& Olofsson, H. 1997, $A \& A$, 318, 535 


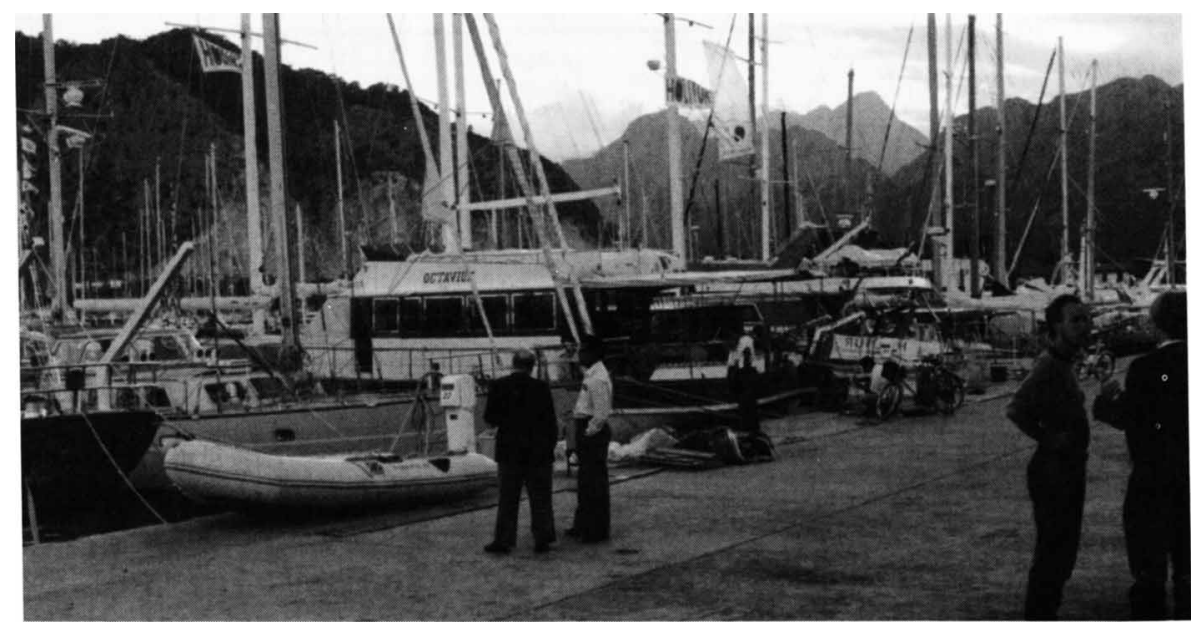

Marina at the site of the conference banquet. 